\title{
Mediastinal Yolk Sac Tumor
}

National Cancer Institute

\section{Source}

National Cancer Institute. Mediastinal Yolk Sac Tumor. NCI Thesaurus. Code C6443.

An extragonadal non-seminomatous malignant germ cell tumor that arises from the mediastinum. It is characterized by the presence of small pale cells with small amount of cytoplasm and round to oval nuclei with small nucleoli forming a variety of patterns, including microcystic, macrocystic, pseudopapillary, myxomatous, hepatoid, polyvesicular vitelline, and solid. It manifests with respiratory distress, thoracic pain, fever, and superior vena cava syndrome. 